\title{
Reply to the Letter to the Editor: Comment on "COVID-19 infection control protocol inside computed tomography suites"
}

\author{
Tsuneo Yamashiro $^{1}$ - Daisuke Utsunomiya ${ }^{1}$ D
}

Received: 21 April 2020 / Accepted: 23 April 2020 / Published online: 4 May 2020

(c) Japan Radiological Society 2020

We appreciate the interest of Dr. Orsi et al. in our report [1], and we respect the valuable comments made by our colleagues from Milan, Italy, as they struggle to combat the effects of the COVID-19 pandemic. Hospitals in central Japan are also facing a similar situation, and therefore, the comments made by them are invaluable.

We decided to use a CT suite as a dedicated room. Our central radiology section has three CT scanners along a corridor with an arrangement to increase the efficiency of scanning and to avoid the crowding of the patients in front of the CT suites while minimizing contact between COVID-19 and non-COVID-19 patients.

The scanners use 0.75 -mm section CT images for the lung parenchyma, and we strongly agree with their reconstruction protocol that comprises thinner section thickness. Dr. Iwasawa also suggested the importance of ultra-high-resolution CT to scrutinize imaging findings of COVID-19 pneumonia [2]. We adopted 2-mm section thickness at the time of submission of our report [1], and since then, this has been changed to $1 \mathrm{~mm}$.

We appreciate their comments regarding contrastenhanced CT use for COVID-19 patients and the importance of support from Healthcare Assistants (HA) in dedicated CT suites.

Thank you again for the important suggestions, and we hope the serious health emergency caused by COVID-19 in Milan improves swiftly.

\section{References}

1. Nakajima K, Kato H, Yamashiro T, Izumi T, Takeuchi I, Nakajima H, et al. COVID-19 pneumonia: infection control protocol inside computed tomography suites. Jpn J Radiol. 2020. https:// doi.org/10.1007/s11604-020-00948-y.

2. Iwasawa T, Sato M, Yamaya T, Sato Y, Uchida Y, Kitamura H, et al. Ultra-high-resolution computed tomography can demonstrate alveolar collapse in novel coronavirus (COVID-19) pneumonia. Jpn J Radiol. 2020. https://doi.org/10.1007/s11604-02000956-y.

Publisher's Note Springer Nature remains neutral with regard to jurisdictional claims in published maps and institutional affiliations.
Daisuke Utsunomiya

d_utsuno@yokohama-cu.ac.jp

1 Diagnostic Radiology, Yokohama City University, 3-9, Fukuura, Kanazawa-ku, Yokohama-shi, Kanagawa 236-0004, Japan 